\title{
THE UNIQUENESS OF MEROMORPHIC FUNCTIONS WITH THEIR DERIVATIVES
}

\author{
QING CAI ZhaNG
}

\begin{abstract}
In this paper, we deal with the problem of uniqueness of meromorphic functions that share one finite value with their derivatives and obtain some theorems which improve a result given by Rainer Brück.
\end{abstract}

\section{Introduction and main results}

By a meromorphic function we shall always mean a function that is meromorphic in the whole complex plane. It is assumed that the reader is familiar with the notations of the Nevanlinna theory such as $T(r, f), m(r, f)$, $N(r, f), \bar{N}(r, f), S(r, f)$ and so on, that can be found, for instance, in [1]. And $N_{1)}(r, 1 / f)$ denotes the counting function of the simple zeros of $f, \bar{N}_{(2}(r, 1 / f)=$ $\bar{N}(r, 1 / f)-N_{1)}(r, 1 / f)$. Let $f$ and $g$ be meromorphic functions and $a$ be a complex constant, we say that $f$ and $g$ share the value $a$ IM (ignoring multiplicity), if $f-a$ and $g-a$ have the same zeros, they share the value $a \mathrm{CM}$ (counting multiplicity), if $f-a$ and $g-a$ have the same zeros with the same multiplicity.

In 1979, E. Mues and N. Steinmetz proved the following theorem in [2].

THEOREM A. Let $f$ be an entire function which is not constant. If $f$ and $f^{\prime}$ share two distinct values $a, b$, then $f^{\prime} \equiv f$.

In 1996, Rainer Brück proved the following in [3]

THEOREM B. Let $f$ be an entire function which is not constant. If $f$ and $f^{\prime}$ share the value $1 C M$, and if $N\left(r, 1 / f^{\prime}\right)=S(r, f)$, then

$$
\frac{f^{\prime}-1}{f-1} \equiv c
$$

for some non-zero constant $c$.

1991 Mathematics Subject Classification. Primary 30D35, Secondary 30D30, 30D20

Key words. meromorphic function, share CM

Received October 13, 1997. 
In this paper, we prove the following results which are improvements of Theorem B.

THEOREM 1. Let $f$ be a non-constant meromorphic function. If $f$ and $f^{\prime}$ share the value $1 C M$, and if

$$
\bar{N}(r, f)+N\left(r, \frac{1}{f^{\prime}}\right)<(\lambda+o(1)) T\left(r, f^{\prime}\right)
$$

for some real constant $\lambda \in(0,1 / 2)$, then $f$ and $f^{\prime}$ satisfy (1).

Remark 1. It is clear that if $f$ and $f^{\prime}$ satisfy the condition of Theorem 1, then $f=A e^{c z}+1-(1 / c)$, where $A, c$ are non-zero constants. And obviously the condition is necessary.

THEOREM 2. Let $f$ be a non-constant meromorphic function, $k$ be a positive integer. If $f$ and $f^{(k)}$ share the value $1 C M$, and if

$$
2 \bar{N}(r, f)+\bar{N}\left(r, \frac{1}{f^{\prime}}\right)+N\left(r, \frac{1}{f^{(k)}}\right)<(\lambda+o(1)) T\left(r, f^{(k)}\right)
$$

for some real constant $\lambda \in(0,1)$, then

$$
\frac{f^{(k)}-1}{f-1} \equiv c
$$

for some non-zero constant $c$.

Remark 2. It is easy to see from (4) that if $f$ and $f^{(k)}$ satisfy the condition of Theorem 2, then $f=A e^{\mu z}+1-1 / c$, where $A, c$ are non-zero constants, and $\mu$ is any $k$-th roots of $c$. And the condition is necessary.

From Theorem 2, we can obtain the following corollaries:

COROLlaRY 1. Let $f$ be a non-constant meromorphic function, $k$ be a positive integer. If $f$ and $f^{(k)}$ share the value $1 C M$, and if

$$
2 \bar{N}(r, f)+\bar{N}\left(r, \frac{1}{f^{\prime}}\right)+N\left(r, \frac{1}{f^{(k)}}\right)<(\lambda+o(1)) T(r, f)
$$

for some real constant $\lambda \in(0,2 / 5)$, then $f$ and $f^{(k)}$ satisfy (4).

COROLlaRY 2. Let $f$ be a non-constant meromorphic function, $k$ be a positive integer. If $f$ and $f^{(k)}$ share the value $1 C M$, and if

$$
(k+1) \bar{N}(r, f)+2 N\left(r, \frac{1}{f^{\prime}}\right)<(\lambda+o(1)) T(r, f)
$$

for some real constant $\lambda \in(0,2 / 5)$, then $f$ and $f^{(k)}$ satisfy (4). 
COROLlary 3. Let $f$ be a non-constant entire function, $k$ be a positive integer. If $f$ and $f^{(k)}$ share the value $1 C M$, and if

$$
\bar{N}\left(r, \frac{1}{f^{\prime}}\right)<(\lambda+o(1)) T(r, f)
$$

for some real constant $\lambda \in(0,1 / 4)$, then $f$ and $f^{(k)}$ satisfy (4).

Obviously Theorem B is included in Corollary 3.

Remark 3. Factly, the real constant $\lambda \in(0,2 / 5)$ in Corollary 1 and Corollary 2 can be stated $\lambda \in(0,(k+1) /(2 k+3))$ instead, that is easy to see from the following proving.

\section{Proof of main results}

The following two lemmas are neened in the following proving.

LEMMA 1 (see [4]). Let $f$ be a non-constant meromorphic function, $k$ be $a$ positive integer, then

$$
N\left(r, \frac{1}{f^{(k)}}\right)<N\left(r, \frac{1}{f}\right)+k \bar{N}(r, f)+S(r, f) .
$$

LEMMA 2. Let $f$ be a non-constant meromorphic function, $k$ be a positive integer, if $f$ and $f^{(k)}$ share the value $1 C M$, then

$$
T(r, f)<\left(2+\frac{1}{k+1}\right) T\left(r, f^{(k)}\right)+S(r, f) .
$$

Especially when $f$ is an entire function, then

$$
T(r, f)<2 T\left(r, f^{(k)}\right)+S(r, f) .
$$

Proof. By Milloux inequality (see [1] or [5]) for $f-1$, we have

$$
T(r, f)<\bar{N}(r, f)+\bar{N}\left(r, \frac{1}{f-1}\right)+\bar{N}\left(r, \frac{1}{f^{(k)}-1}\right)+S(r, f) .
$$

Since $f$ and $f^{(k)}$ share the value $1 \mathrm{CM}$, and

$$
T\left(r, f^{(k)}\right)>N\left(r, f^{(k)}\right)>(k+1) \bar{N}(r, f)
$$

so (8) holds. (9) can be got immediately from (10). This lemma is thus proved.

Now we turn to prove the theorems. Theorem 1 is the particular case of Theorem 2, so we need only to prove Theorem 2. Define

$$
F=\frac{f^{(k+2)}}{f^{(k+1)}}-\frac{f^{\prime \prime}}{f^{\prime}}-2 \frac{f^{(k+1)}}{f^{(k)}-1}+2 \frac{f^{\prime}}{f-1} .
$$


Firstly assume that $F \not \equiv 0$, then $m(r, f)=S(r, f) . \quad N_{0}\left(r, 1 / f^{(k+1)}\right)$ denotes the counting function corresponding the zeros of $f^{(k+1)}$ which are not the zeros of $f^{\prime}, f^{(k)}$ and $f^{(k)}-1$ with the multiple zeros are counted multiplicity times, $\bar{N}_{0}\left(r, 1 / f^{(k+1)}\right)$ denotes that case the multiple zeros are only counted one time. Since $f$ and $f^{(k)}$ share the value $1 \mathrm{CM}$, it is easy to see by calculating that the zeros of $f-1$ are not the poles of $F$, so we have

$$
N(r, F) \leq \bar{N}(r, f)+\bar{N}\left(r, \frac{1}{f^{\prime}}\right)-\bar{N}_{(2}\left(r, \frac{1}{f-1}\right)+\bar{N}_{(2}\left(r, \frac{1}{f^{(k)}}\right)+\bar{N}_{0}\left(r, \frac{1}{f^{(k+1)}}\right) .
$$

And noticing that $m(r, F)=S(r, f)$ and

$$
\bar{N}_{(2}\left(r, \frac{1}{f-1}\right)=\bar{N}_{(2}\left(r, \frac{1}{f^{(k)}-1}\right)
$$

then

$$
\begin{aligned}
T(r, F) \leq & \bar{N}(r, f)+\bar{N}\left(r, \frac{1}{f^{\prime}}\right)+\bar{N}_{(2}\left(r, \frac{1}{f^{(k)}}\right)+\bar{N}_{0}\left(r, \frac{1}{f^{(k+1)}}\right) \\
& -\bar{N}_{(2}\left(r, \frac{1}{f^{(k)}-1}\right)+S(r, f) .
\end{aligned}
$$

By calculating it can be shown that the simple zeros of $f-1$ are the zeros of $F$. And as $f$ and $f^{(k)}$ share the value $1 \mathrm{CM}$, we have

$$
N_{1)}\left(r, \frac{1}{f-1}\right)=N_{1)}\left(r, \frac{1}{f^{(k)}-1}\right) \leq N\left(r, \frac{1}{F}\right) \leq T(r, F)+O(1) .
$$

Combining (11) and (12) we obtain

$$
\begin{aligned}
& \bar{N}\left(r, \frac{1}{f^{(k)}-1}\right)=N_{1)}\left(r, \frac{1}{f^{(k)}-1}\right)+\bar{N}_{(2}\left(r, \frac{1}{f^{(k)}-1}\right) \\
& \quad \leq \bar{N}(r, f)+\bar{N}\left(r, \frac{1}{f^{\prime}}\right)+\bar{N}_{(2}\left(r, \frac{1}{f^{(k)}}\right)+\bar{N}_{0}\left(r, \frac{1}{f^{(k+1)}}\right)+S(r, f)
\end{aligned}
$$

By using the second fundamental theorem for $f^{(k)}$, we have

$$
\begin{aligned}
T\left(r, f^{(k)}\right)< & \bar{N}(r, f)+\bar{N}\left(r, \frac{1}{f^{(k)}}\right)+\bar{N}\left(r, \frac{1}{f^{(k)}-1}\right) \\
& -N_{0}\left(r, \frac{1}{f^{(k+1)}}\right)+S\left(r, f^{(k)}\right) .
\end{aligned}
$$

From Lemma 2 we can get $S(r, f)=S\left(r, f^{(k)}\right)$. Combining this and (13) and (14) and

$$
\bar{N}\left(r, \frac{1}{f^{(k)}}\right)+\bar{N}_{(2}\left(r, \frac{1}{f^{(k)}}\right) \leq N\left(r, \frac{1}{f^{(k)}}\right)
$$


we get

$$
T\left(r, f^{(k)}\right)<2 \bar{N}(r, f)+\bar{N}\left(r, \frac{1}{f^{\prime}}\right)+N\left(r, \frac{1}{f^{(k)}}\right)+S(r, f) .
$$

By (15) and (3) we have $T\left(r, f^{(k)}\right)=S(r, f)$, and from (8) we conclude $T(r, f)=S(r, f)$ which is a contradiction.

Therefore, we have $F \equiv 0$, and integration yields

$$
C \frac{f^{(k+1)}}{f^{\prime}} \equiv\left(\frac{f^{(k)}-1}{f-1}\right)^{2}
$$

where $C$ is a non-zero constant.

We assume that $f^{(k+1)} / f^{\prime}$ is not constant. From (16) and that $f$ and $f^{(k)}$ sharing the value $1 \mathrm{CM}$, it is clear that $f^{(k+1)} / f^{\prime}$ has no zeros and no poles, so

$$
T\left(r, \frac{f^{(k+1)}}{f^{\prime}}\right)=m\left(r, \frac{f^{(k+1)}}{f^{\prime}}\right)=S(r, f) .
$$

If $z_{0}$ is a simple zero of $f-1$, we know by calculating that $\left(f^{(k)}-1\right) /\left.(f-1)\right|_{z=z_{0}}=f^{(k+1)}\left(z_{0}\right) / f^{\prime}\left(z_{0}\right)$, and from $(16)$ thus $f^{(k+1)}\left(z_{0}\right) /$ $f^{\prime}\left(z_{0}\right)=C$, so $z_{0}$ is also the zero of $\left(f^{(k+1)} / f^{\prime}\right)-C$, then

$$
N_{1)}\left(r, \frac{1}{f-1}\right) \leq N\left(r, \frac{1}{\frac{f^{(k+1)}}{f^{\prime}}-C}\right) \leq T\left(r, \frac{f^{(k+1)}}{f^{\prime}}\right)+O(1)=S(r, f)
$$

therefore

$$
\begin{aligned}
\bar{N}\left(r, \frac{1}{f^{(k)}-1}\right) & =\bar{N}\left(r, \frac{1}{f-1}\right)=N_{1)}\left(r, \frac{1}{f-1}\right)+\bar{N}_{(2}\left(r, \frac{1}{f-1}\right) \\
& \leq \bar{N}\left(r, \frac{1}{f^{\prime}}\right)+S(r, f)
\end{aligned}
$$

this implies that (13) still holds. Similarly we can get the contradition $T(r, f)=$ $S(r, f)$ again. Then $f^{(k+1)} / f^{\prime}$ is a constant, and so the proof of Theorem 2 is finished.

Corollary 1 can be obtained by Theorem 2 and (8).

By Lemma 1 we get

$$
N\left(r, \frac{1}{f^{(k)}}\right)<N\left(r, \frac{1}{f^{\prime}}\right)+(k-1) \bar{N}(r, f)+S(r, f)
$$

from this and Corollary 1 we obtained Corollary 2 .

Corollary 3 can be obtained by Theorem 2 and (9) and (17).

Acknowledgement. The author appreciates Professor Hong-Xun Yi for his helpful direction. 


\section{REFERENCES}

[1] W. K. Hayman, Meromorphic Function, Clarendon Press, Oxford, 1964.

[2] E. Mues and N. Steinmetz, Meromorphe Funktionen, die mit ihrer Ableitung Werte teilen, Manuscripta Math., 29 (1979), 195-206.

[3] RAINER BRÜCK, On entire functions which share one value CM with their first derivative, Results in Math., 30 (1996), 21-24.

[4] Hong-XuN Yi, Unıqueness of meromorphic functions and a question of C. C. Yang, Complex Variables Theory Appl., 14 (1990), 169-176.

[ 5 ] H. Milloux, Extension d'un théorème de M. R. Nevanlinna et applications, Act. Scient. et Ind. No. 888, 1940.

Department of Basic Courses

Shandong Finance Institute

JiNAN, SHANDONG 250014

People's RePUBlic of China 